Pacific

Journal of

Mathematics

EXISTENCE AND EXPLICIT CONSTRUCTIONS OF HCMU METRICS ON $S^{2}$ AND $T^{2}$

QING CHEN AND YINGYi Wu

Volume $240 \quad$ No. 2

April 2009 


\title{
EXISTENCE AND EXPLICIT CONSTRUCTIONS OF HCMU METRICS ON $S^{2}$ AND $T^{2}$
}

\author{
QING ChEn AND YingYi Wu
}

\begin{abstract}
We prove the existence of HCMU metrics on $S^{2}$ and $T^{2}$ with explicit constructions and the solution of appropriate ODEs.
\end{abstract}

\section{Introduction}

The classical uniformization theorem says that on a compact Riemannian surface without boundary there is a constant curvature metric in any conformal class of metrics. There have been many attempts to generalize this theory to surfaces with boundary. The main focus, started by the independent work of Troyanov [1991] and McOwen [1988], has been to study the existence or nonexistence of constant curvature metrics on surfaces with conical singularities. But in general one should not expect to get a clear-cut statement about the existence (or nonexistence) of solutions, since the constant curvature equation is overdetermined in this case. Therefore we consider a wider class of metrics, namely extremal metrics, as the generalization of constant curvature metrics on Riemannian surfaces with conical singularities.

Let $M$ be any compact, oriented smooth Riemannian surface without boundary and let $P:=\left\{p_{1}, p_{2}, \ldots, p_{n}\right\}$ be a finite set of points on $M$. Let $g_{0}$ be a smooth metric on $M \backslash P$ for which, in a neighborhood of each $p_{i}$, there exists a local complex coordinate chart $(U, z)$ with $z\left(p_{i}\right)=0$ such that

$$
\left.g_{0}\right|_{U}=h(z, \bar{z}) \frac{1}{|z|^{2-2 \alpha_{i}}}|d z|^{2},
$$

where $h: U \rightarrow \mathbb{R}$ is a continuous positive function that is smooth on $U \backslash\{0\}$, that is, $g_{0}$ has a conical singularity at $p_{i}$ with the angle $2 \pi \alpha_{i}$ (with $\alpha_{i}>0$ ). Then denote the K-surface associated to $M$ by $M_{\left\{\alpha_{1}, \alpha_{2}, \ldots, \alpha_{n}\right\}}$.

MSC2000: primary 32C15, 53C21, 53C25, 53C55, 58D17; secondary 53C25, 32C15, 53C55, 53C21, $58 \mathrm{D} 17$.

Keywords: Calabi energy, HCMU metric, Riemannian surface.

Chen is supported by NSFC Grant 10871184. 
Next we consider the conformal class of $g_{0}$, defined by

$$
\mathscr{S}\left(g_{0}\right)=\left\{g=e^{2 \varphi} g_{0}, \varphi \in H_{0}^{2,2}(M \backslash P) \mid \int_{M \backslash P} e^{2 \varphi} d g_{0}=\int_{M \backslash P} d g_{0}\right\},
$$

which is the conformal class of $g_{0}$ such that each of $\mathscr{Y}\left(g_{0}\right)$ has the same area as $g_{0}$ and coincides with $g_{0}$ on a neighborhood of singularities. Define the Calabi energy functional in $\mathscr{S}\left(g_{0}\right)$ by $E(g)=\int_{M \backslash P} K^{2} d g$, where $K$ is the Gaussian curvature of the metric $g$. The Euler-Lagrange equation of $E(g)$ is [Chen 2000]

$$
\Delta_{g} K+K^{2}=C,
$$

where $C$ is a constant; equivalently, on a local complex coordinate chart of a regular point, it is

$$
\frac{\partial}{\partial \bar{z}} K_{, z z}=0
$$

where $K_{, z z}$ is the second order $(0,2)$ covariant derivative of $K$.

A metric that satisfies (2) or (3) is called an extremal metric. Equation (3) has two special cases; one is

$$
K \equiv \text { const }
$$

and another is

$$
K_{, z z}=0 \quad \text { with } K \neq \text { const. }
$$

A metric that satisfies (5) is called an HCMU metric (from "the Hessian of the curvature of the metric is umbilical"; see [Chen 2000]). Throughout this paper, we assume that an HCMU metric is of finite area and finite Calabi energy.

For $p \in M \backslash P$ we assume that $(U, z)$ is a complex coordinate chart around $p(z(p)=0)$. Then $g$ can be written as

$$
g=e^{2 \varphi(z, \bar{z})}|d z|^{2} \quad \text { with } K=-\triangle \varphi / e^{2 \varphi},
$$

and the HCMU metric of Equation (5) can be written as

$$
K_{, z z}=\frac{\partial^{2} K}{\partial z^{2}}-2 \frac{\partial K}{\partial z} \frac{\partial \varphi}{\partial z}=0 .
$$

Since the gradient vector field $\nabla K$ is

$$
\nabla K=\sqrt{-1} K^{\prime z} \frac{\partial}{\partial z}=\sqrt{-1} e^{-2 \varphi} \frac{\partial K}{\partial \bar{z}} \frac{\partial}{\partial z},
$$

Equation (7) implies that $\nabla K$ is holomorphic, or equivalently, its real part

$$
\vec{V}=\frac{1}{2}\left(\sqrt{-1} K^{\prime z} \frac{\partial}{\partial z}-\sqrt{-1} K^{\prime \bar{z}} \frac{\partial}{\partial \bar{z}}\right)
$$


is a Killing vector field and the integral curve of $\vec{V}$ is the level set of the function $K$.

In [2000], X.X. Chen studied the flow of the Killing field $\vec{V}$ and obtained the following obstruction theorem.

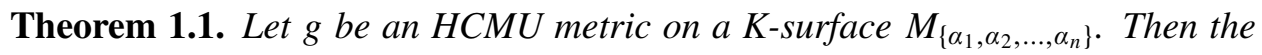
Euler character of the underlying surface should be determined by

$$
\chi(M)=\sum_{i=1}^{j}\left(1-\alpha_{i}\right)+(n-j)+s,
$$

where $s$ is the number of critical points of the curvature $K$ (excluding the singular points of $g$ ). Here we assume that $\alpha_{1}, \alpha_{2}, \ldots, \alpha_{k}$ for $0 \leq k \leq n$ are the only integers in the set of prescribed angles $\alpha_{1}, \alpha_{2}, \ldots, \alpha_{n}$; we also assume that $p_{j+1}, p_{j+2}, \ldots, p_{k}$ are the only local extremal points of $K$ in the set of singular points $\left\{p_{j}: 0 \leq j \leq k\right\}$.

Indeed, the curvature $K$ of an HCMU metric can be continuously extended to singular points, and the corresponding vector field $\vec{V}$ has only finite singularities. On the other hand, each singular point $p_{j}$ for $1 \leq j \leq n$ is either an extremal point of $K$ or a saddle point of $K$. In the latter case the corresponding singular angle $\alpha_{j}$ is always an integer, and the index of $\vec{V}$ at the point is $1-\alpha_{j}$. See [Chen 2000].

Remark 1.1. By (10), if there is no saddle point of $K$ on $M$, the genus of the underlying surface must be zero. In this case an HCMU metric is rotationally symmetric, and the surface is called a football (see [Chen et al. 2005; Chen 2000]). Therefore we will henceforth assume that the number of the saddle points of $K$ is greater than zero.

Further, in [Chen et al. 2005], X.X. Chen and the authors of this paper studied the global structure of HCMU metrics and obtained a decomposition theorem:

Theorem 1.2. Let $g$ be an HCMU metric on a K-surface of $M$. Then there are a finite number of geodesics that connect the extremal points and the saddle points of the curvature $K$ together. In fact, $M$ can be divided into a finite number of pieces by cutting along these geodesics, and each piece is locally isometric to a football.

In [2002], C.S. Lin and X.H. Zhu studied $\nabla K$ and constructed a class of socalled exceptional HCMU metrics on $S^{2}$. These metrics have finite conical singular angles of the form $2 \pi n$, with $n$ an integer, and have singularities exclusively at the saddle points of $K$.

Theorem 1.3. Let $p_{1}=\infty$ and $p_{i}=z_{i}$ for $i=2, \ldots, n$ be $n$ points on $S^{2}=\mathbb{C} \cup\{\infty\}$, and let $\alpha_{1} \geq 2, \ldots, \alpha_{n} \geq 2$ be $n$ positive integers. Let

$$
\alpha=\alpha_{1}+\sum_{i=2}^{n}\left(\alpha_{i}-1\right) .
$$


Then $g=e^{2 \varphi}|d z|^{2}$ is an exceptional HCMU metric with the conical singular angle $2 \pi \alpha_{i}$ at $p_{i}$ on $S^{2}$ if and only if there are a positive integer $k$, a complex number $B \neq 0$, and a degree $(\alpha+1)$ polynomial $f(z)$ on $\mathbb{C}$ with distinct roots $\gamma_{l}$, with the properties that $3 k \leq \alpha+1$ and that the $\gamma_{l}$ satisfy

$$
\sum_{l=1}^{k} \frac{-2 a}{z-\gamma_{l}}+\sum_{l^{\prime}=k+1}^{\alpha+1} \frac{2}{z-\gamma_{l^{\prime}}}=\frac{B \prod_{i=2}^{n}\left(z-z_{i}\right)^{\alpha_{i}-1}}{\prod_{l=1}^{\alpha+1}\left(z-\gamma_{l}\right)},
$$

where $a=(\alpha+1-k) / k$. Furthermore, $K$ and $\varphi$ are given by

$$
\frac{1}{\left(K+\frac{(a-2) \beta}{2 a-1}\right)^{a}}(\beta-K)\left(K+\frac{\beta(a+1)}{2 a-1}\right)^{a-1}=A \prod_{l=1}^{k}\left|z-\gamma_{l}\right|^{-2 a} \prod_{l^{\prime}=k+1}^{\alpha+1}\left|z-\gamma_{l^{\prime}}\right|^{2},
$$

and

$$
\varphi=\frac{1}{2} \ln \left(\left(1 /|F(z)|^{2}\right)\left(-\frac{1}{3} K^{3}+c K+c^{\prime}\right)\right),
$$

where $A>0$ is some constant and

$$
\begin{aligned}
\beta & =\sqrt{c}(2 a-1) / \sqrt{a^{2}-a+1}, \\
c^{\prime} & =\frac{(a+1)(a-2) \beta^{3}}{3(2 a-1)^{2}}, \\
F(z) & =\left(z-z_{2}\right)^{-\left(a_{2}-1\right)} \cdots\left(z-z_{n}\right)^{-\left(\alpha_{n}-1\right)} f(z), \\
f(z) & =B^{-1} \frac{-3 a(a-1) \beta^{2}}{(2 a-1)^{2}} \prod_{l=1}^{\alpha+1}\left(z-\gamma_{l}\right) .
\end{aligned}
$$

In this paper we generalize Theorem 1.3 to nonexceptional metrics, and get existence theorems for HCMU metrics on $S^{2}$ and $T^{2}$.

Theorem 1.4. Let $S^{2}=\mathbb{C} \cup\{\infty\}$ with $p_{1}=\infty, p_{2}=z_{2}, \ldots, p_{n}=z_{n}$. Suppose $\alpha_{1}, \ldots, \alpha_{n}$ are positive real numbers and $\alpha_{1}, \ldots, \alpha_{j}$ for $j>0$ are integers with $\alpha_{i^{\prime}} \geq 2$ for $i^{\prime}=1, \ldots, j$. Then the statements that

- there exists an HCMU metric on $S^{2}$ such that $p_{1}, p_{2}, \ldots, p_{n}$ are the only singularities of the metric, with corresponding singular angles $2 \pi \alpha_{1}, 2 \pi \alpha_{2}$, $\ldots, 2 \pi \alpha_{n}$, and

- $p_{1}, p_{2}, \ldots, p_{j}$ are the only saddle points of $K$

are equivalent to the statements that

- $s=2+\sum_{i^{\prime}=1}^{j} \alpha_{i^{\prime}}-n \geq 0$, and

- there are a permutation $\delta$ of $j+1, \ldots, n$, an integer $l$ with $0 \leq l \leq n-j$, an integer $t$ with $0 \leq t \leq s$, and $\left\{\beta_{1}, \ldots, \beta_{s}\right\} \subset \mathbb{C} \backslash\left\{z_{2}, \ldots, z_{n}\right\}$ such that

$$
\alpha_{\delta(1)}+\alpha_{\delta(2)}+\cdots+\alpha_{\delta(l)}+t>\alpha_{\delta(l+1)}+\cdots+\alpha_{\delta(n-j)}+s-t
$$


and

$$
\begin{aligned}
\sum_{k=1}^{l} \frac{\alpha_{\delta(k)}}{z-z_{\delta(k)}}+\sum_{k^{\prime}=l+1}^{n-j} \frac{(a-1) \alpha_{\delta\left(k^{\prime}\right)}}{z-z_{\delta\left(k^{\prime}\right)}}+\sum_{m=1}^{t} \frac{1}{z-\beta_{m}}+\sum_{m^{\prime}=t+1}^{s} \frac{a-1}{z-\beta_{m^{\prime}}}= \\
\frac{B \prod_{i^{\prime}=2}^{j}\left(z-z_{i^{\prime}}\right)^{\alpha_{i^{\prime}}-1}}{\prod_{k=j+1}^{n}\left(z-z_{k}\right) \prod_{m=1}^{s}\left(z-\beta_{m}\right)}
\end{aligned}
$$

where $B$ is a constant and $a-1=-\alpha_{\max } / \alpha_{\min }$. Here, we let

$$
\begin{aligned}
& \alpha_{\max }=\alpha_{\delta(1)}+\alpha_{\delta(2)}+\cdots+\alpha_{\delta(l)}+t, \\
& \alpha_{\min }=\alpha_{\delta(l+1)}+\cdots+\alpha_{\delta(n-j)}+s-t .
\end{aligned}
$$

When the underlying Riemannian surface $M$ is a torus $T^{2}$, we have an existence theorem similar to Theorem 1.4, but there are some differences due to the representations of elliptic functions. The complex structure of $T^{2}$ is determined up to transformations in $\operatorname{SL}(2, \mathbb{Z})$ by a pair $\left(w_{1}, w_{2}\right)$ with $w_{1}, w_{2} \in \mathbb{C}$ and $w_{1} / w_{2} \notin \mathbb{R}$. Let $\Gamma:=\mathbb{Z} w_{1}+\mathbb{Z} w_{2}$ be the lattice spanned by $w_{1}$ and $w_{2}$. Then $T^{2}=\mathbb{C} / \Gamma$. We call $w_{1}$ and $w_{2}$ elementary periods, and $P_{z}=\left\{z+x w_{1}+y w_{2} \mid z \in \mathbb{C}, x \in[0,1), y \in[0,1)\right\}$ an elementary parallelogram with base point $z$. A meromorphic function on $T^{2}$, or equivalently a meromorphic function with double periods on $\mathbb{C}$, is an elliptic function. Any elliptic function can be expressed by Weierstrass $\sigma$ functions or Weierstrass $\zeta$ functions [Lang 1987], where

$$
\zeta(z):=\frac{1}{z}+\sum_{\substack{w \in \Gamma, w \neq 0}}\left(\frac{1}{z-w}+w+\frac{z}{w^{2}}\right), \quad \sigma(z):=z \prod_{\substack{w \in \Gamma \\ w \neq 0}}\left(1-\frac{z}{w}\right) e^{z / w+\frac{1}{2} z^{2} / w^{2}} .
$$

Theorem 1.5. Let $T^{2}=\mathbb{C} / \Gamma$ and $P_{\mathrm{o}}$ be the elementary parallelogram with base point the origin, and let $p_{1}=z_{1}, \ldots, p_{n}=z_{n}$ on $P_{0}$. Suppose $\alpha_{1}, \ldots, \alpha_{n}$ are positive real numbers and $\alpha_{1}, \ldots, \alpha_{j}$ are integers with $\alpha_{i^{\prime}} \geq 2$ for $i^{\prime}=1, \ldots, j$. Then the statements that

(1) there exists an HCMU metric on $T^{2}$ such that $p_{1}, \ldots, p_{n}$ are the only singularities of the metric, with corresponding singular angles $2 \pi \alpha_{1}, \ldots, 2 \pi \alpha_{n}$, and

(2) $p_{1}, \ldots, p_{j}$ are the only saddle points of $K$

are equivalent to the statements that

(1) $s=\sum_{i^{\prime}=1}^{j} \alpha_{i^{\prime}}-n \geq 0$, and

(2) there are a permutation $\delta$ of $j+1, \ldots, n$, an integer $l, 0 \leq l \leq n-j$, an integer $t$ with $0 \leq t \leq s$ and a set $\left\{\beta_{1}, \ldots, \beta_{s}\right\} \subset P_{\mathrm{o}} \backslash\left\{z_{1}, \ldots, z_{n}\right\}$ such that

$$
\alpha_{\delta(1)}+\alpha_{\delta(2)}+\cdots+\alpha_{\delta(l)}+t>\alpha_{\delta(l+1)}+\cdots+\alpha_{\delta(n-j)}+s-t
$$


and

- there exist $n_{1}, n_{2} \in \mathbb{Z}$ such that

$$
\begin{aligned}
& \sum_{i^{\prime}=1}^{j}\left(\alpha_{i^{\prime}}-1\right) z_{i^{\prime}}-\sum_{k=j+1}^{n} z_{k}-\sum_{m=1}^{s} \beta_{m}=n_{1} w_{1}+n_{2} w_{2}, \\
& \lambda+\sum_{k=1}^{l} \alpha_{\delta(k)} \zeta\left(z-z_{\delta(k)}\right)+\sum_{k^{\prime}=l+1}^{n-j}(a-1) \alpha_{\delta\left(k^{\prime}\right)} \zeta\left(z-z_{\delta\left(k^{\prime}\right)}\right) \\
& +\sum_{m=1}^{t} \zeta\left(z-\beta_{m}\right)+\sum_{m^{\prime}=t+1}^{s}(a-1) \zeta\left(z-\beta_{m^{\prime}}\right) \\
& =\frac{B \prod_{i^{\prime}=1}^{j} \sigma\left(z-z_{i^{\prime}}\right)^{\alpha_{i^{\prime}}-1}}{\prod_{k=j+1}^{n} \sigma\left(z-z_{k}\right) \prod_{m=1}^{s-1} \sigma\left(z-\beta_{m}\right) \sigma\left(z-\beta_{s}-n_{1} w_{1}-n_{2} w_{2}\right)},
\end{aligned}
$$

where $B$ is a constant, $a-1=-\alpha_{\max } / \alpha_{\min }$, (with $\alpha_{\max }$ and $\alpha_{\min }$ as in (12)) and

$$
\lambda=\frac{1}{w_{1} \bar{w}_{2}-w_{2} \bar{w}_{1}}\left(\operatorname{Re}\left[\eta_{1} \rho\right] \bar{w}_{2}-\operatorname{Re}\left[\eta_{2} \rho\right] \bar{w}_{1}\right)
$$

with $\eta_{1}=2 \zeta\left(w_{1} / 2\right), \eta_{2}=2 \zeta\left(w_{2} / 2\right)$, and

$$
\rho=2\left(\sum_{k=1}^{l} \alpha_{\delta(k)} z_{\delta(k)}+\sum_{k^{\prime}=l+1}^{n-j}(a-1) \alpha_{\delta\left(k^{\prime}\right)} z_{\delta\left(k^{\prime}\right)}+\sum_{m=1}^{t} \beta_{m}+\sum_{m^{\prime}=t+1}^{s}(a-1) \beta_{m^{\prime}}\right) .
$$

\section{Local behavior of an HCMU metric}

Let $g=e^{2 \varphi}|d z|^{2}$ be an HCMU metric on a domain $(\Omega, z)$ of $M$, let $K$ be the Gaussian curvature, and let $\vec{V}$ be the real part of $\nabla K$. When $\Omega$ does not contain the singularities of $\vec{V}$, we let $F(z)=e^{-2 \varphi} K_{\bar{z}}$. Then $F$ is a holomorphic function that does not vanish on $\Omega$. By (2) on $\Omega$ we have

$$
K_{z \bar{z}}=\left(C-K^{2}\right) e^{2 \varphi}=\left(\frac{C K-\frac{1}{3} K^{3}}{F}\right)_{\bar{z}} .
$$

Then

$$
\begin{aligned}
K_{z} & =\frac{-\frac{1}{3} K^{3}+C K+C^{\prime}}{F}, \\
e^{2 \varphi} & =\frac{-\frac{1}{3} K^{3}+C K+C^{\prime}}{|F|^{2}},
\end{aligned}
$$

where $C^{\prime} \in \mathbb{R}$. If $p$ is a singular point of $\vec{V}$ and $\left(\Omega_{p}, z\right)$ is a local complex coordinate chart around $p$ with $z(p)=0$, then (15), (16) and (17) hold on $\Omega_{p} \backslash\{0\}$, and $F$ is a meromorphic function on $\Omega_{p}$. 
Proposition 2.1 [Lin and Zhu 2002]. Let $g=e^{2 \varphi}|d z|^{2}$ be an HCMU metric on $\Omega_{p} \backslash\{0\}$ with a singular angle $2 \pi \alpha$ at the origin. Then near the origin, $F(z)$ has the expansion

$$
F(z)= \begin{cases}z g(z) & \text { if } \alpha \text { is not an integer } \\ z^{-(\alpha-1)}(c+g(z)) \text { or } z g(z) & \text { if } \alpha \text { is an integer }\end{cases}
$$

where $c \neq 0$ a constant and $g(z)$ is some holomorphic function on $\Omega_{p}$.

We refine Proposition 2.1 as the following:

Proposition 2.2. Let $g=e^{2 \varphi}|d z|^{2}$ be an HCMU metric on $\Omega_{p} \backslash\{0\}$ with a singular angle $2 \pi \alpha$ at the origin. Then near the origin, $F(z)$ has the expansion

$$
F(z)= \begin{cases}z g(z) & \text { if } z=0 \text { is a local extremal point of } K, \\ z^{-(\alpha-1)} g(z) & \text { if } z=0 \text { is a saddle point of } K,\end{cases}
$$

where $g(z)$ is a holomorphic function on $\Omega_{p}$ with $g(0) \neq 0$.

Proof. If $p$ is a local extremal point of $K$, we may assume without loss of generality that $p$ is the local maximum point of $K$. Since $\vec{V}$ is a Killing vector field and the integral curves of $\vec{V}$ in a neighborhood of $p$ are all circles centered at $p$ (see [Chen 2000]), near $p$ we have

$$
g=d u^{2}+f(u)^{2} d \theta^{2} \quad \text { for } 0 \leq u \leq \epsilon, 0 \leq \theta \leq 2 \pi,
$$

with $f(u)>0$ for $u>0, f(0)=0, f^{\prime}(0)=\alpha$, and $\epsilon$ a small real number. That $g$ is an HCMU metric implies that $d K / d u=c f$, where $c$ is a negative constant. Let $v=-\int_{u}^{\epsilon} d s / f$. Then $g=f^{2}\left(d v^{2}+d \theta^{2}\right)$ and $\lim _{u \rightarrow 0} v=-\infty$. Thus if we let $z^{\prime}=v+\sqrt{-1} \theta$ and $w=e^{z^{\prime}}$, then $w$ is a local complex chart around $p$ with $w(p)=0$. It follows that $g=\left(f^{2} /|w|^{2}\right)|d w|^{2}$ and $K_{\bar{w}}=\frac{1}{2} c f^{2} / \bar{w}$, so $F(w)=\frac{1}{2} c w$. This proves the first part of the proposition.

To prove the remaining case, we first show that if locally a vector field is given by $z^{k} g(z) \frac{\partial}{\partial z}$ with $g(0) \neq 0$ and $k \in \mathbb{Z}$, then the index of its real part at 0 is $k$. Let $z=r \exp (\sqrt{-1} \theta), g(z)=g_{1}+\sqrt{-1} g_{2}$ and $\frac{\partial}{\partial z}=\frac{1}{2}\left(\frac{\partial}{\partial x}-\sqrt{-1} \frac{\partial}{\partial y}\right)$. Then the real part of this vector field is

$$
\frac{1}{2} r^{k}\left(\left(g_{1} \cos k \theta-g_{2} \sin k \theta\right) \frac{\partial}{\partial x}+\left(g_{2} \cos k \theta+g_{1} \sin k \theta\right) \frac{\partial}{\partial y}\right) .
$$

Thus a direct calculation shows that

$$
\text { Index }_{p}=k+\frac{1}{2 \pi} \int_{0}^{2 \pi} \frac{g_{1} \frac{\partial g_{2}}{\partial \theta}-g_{2} \frac{\partial g_{1}}{\partial \theta}}{g_{1}^{2}+g_{2}^{2}} d \theta .
$$

Since $g(0) \neq 0$, we assume without loss of generality that $g_{2}(0) \neq 0$. Then the second term on the right side of (22) is $(2 \pi)^{-1} \int_{0}^{2 \pi} d \arctan \left(g_{1} / g_{2}\right)=0$. 
Now if $p$ is a saddle point of $K$ with a singular angle $2 \pi \alpha$, then $\alpha$ is an integer and the index of $\vec{V}$ at $p$ is $1-\alpha$. By Proposition 2.1, $F(z)=z^{-(\alpha-1)} g(z)$. Therefore by what we have proved above, the index of $\vec{V}$ at $p$ is at least $1-\alpha$, so $g(0) \neq 0$. This proves the second part of Proposition 2.2.

Corollary 2.1. $K$ is smooth at any saddle point of $K$.

Proof. We still use the notations of Proposition 2.2. By Proposition 2.2 on $\Omega_{p} \backslash\{0\}$, $1 / F(z)=z^{(\alpha-1)} / g(z)$, so by (17) $e^{2 \varphi}$ is a continuous function on $\Omega_{p}$. By (16) the first weak derivatives of $K$ exist on $\Omega_{p}$. Hence by (15) $\Delta K \in L^{q}\left(\Omega_{p}\right)$, where $q$ is big enough. Then by the Calderon-Zygmund inequality $K \in W^{2, q}\left(\Omega_{p}^{\prime}\right)$ for $\Omega_{p}^{\prime} \subset \subset \Omega_{p}$, and by the Sobolev embedding theorem $K \in C^{1}\left(\Omega_{p}^{\prime}\right)$. Again by (16) and using a bootstrap technique, we have $K \in C^{\infty}\left(\Omega_{p}^{\prime}\right)$.

\section{Proof of Theorem 1.4}

3.1. Proof of necessity. Let $g=e^{2 \varphi}|d z|^{2}$ be an HCMU metric on $S^{2}=\mathbb{C} \cup\{\infty\}$ with singular points $p_{1}=\infty, p_{2}=z_{2}, \ldots, p_{n}=z_{n}$. If $p_{1}, p_{2}, \ldots, p_{j}$ are the only saddle points of $K$, then by Proposition $2.2 F(z)=e^{-2 \varphi} K_{\bar{z}}$ is a rational function on $\mathbb{C}$ with poles $\infty, z_{2}, \ldots, z_{j}$ and zeros the local extremal points of $K$. Therefore

$$
F(z)=\frac{T \prod_{k=j+1}^{n}\left(z-z_{k}\right) \prod_{m=1}^{s}\left(z-\beta_{m}\right)}{\prod_{i=2}^{j}\left(z-z_{i}\right)^{\alpha_{i}-1}},
$$

where $T$ is a complex number and the smooth extremal points of $K$ are denoted $\beta_{1}, \beta_{2}, \ldots, \beta_{s}$, where $s=\sum_{i^{\prime}=1}^{j}\left(\alpha_{i^{\prime}}-1\right)+2-(n-j)$. By (23) the order of each zero of $F(z)$ is one; thus, there are constants $c_{j+1}, \ldots, c_{n}$ and $c_{1}^{\prime}, \ldots, c_{s}^{\prime}$ such that

$$
\frac{1}{F(z)}=\sum_{k=j+1}^{n} \frac{c_{k}}{z-z_{k}}+\sum_{m=1}^{s} \frac{c_{m}^{\prime}}{z-\beta_{m}}=\frac{1}{T} \frac{\prod_{i^{\prime}=2}^{j}\left(z-z_{i^{\prime}}\right)^{\alpha_{i^{\prime}}-1}}{\prod_{k=j+1}^{n}\left(z-z_{k}\right) \prod_{m=1}^{s}\left(z-\beta_{m}\right)} .
$$

Since $\left\{z_{j+1}, \ldots, z_{n}, \beta_{1}, \ldots, \beta_{s}\right\}$ is the set of all extremal points of $K$, there are an integer $l$ with $0 \leq l \leq n-j$ and a permutation $\delta$ of $j+1, \ldots, n$ such that $z_{\delta(1)}, \ldots, z_{\delta(l)}$ are the local maximum points of $K$ and $z_{\delta(l+1)}, \ldots, z_{\delta(n-j)}$ are the local minimum points of $K$; meanwhile we suppose that there are $t$ smooth local maximum points $\beta_{1}, \ldots, \beta_{t}$ of $K$ with $0 \leq t \leq s$, and $s-t$ smooth local minimum points $\beta_{t+1}, \ldots, \beta_{s}$ of $K$.

On $\mathbb{C} \backslash\left\{z_{2}, \ldots, z_{n}, \beta_{1}, \ldots, \beta_{s}\right\}$, we have since (16) holds this result:

Proposition 3.1. In (16),

$$
-\frac{1}{3} K^{3}+C K+C^{\prime}=-\frac{1}{3}\left(K-K_{1}\right)\left(K-K_{2}\right)\left(K+K_{1}+K_{2}\right),
$$

where $K_{1}=K_{\max }$ and $K_{2}=K_{\min }$. 
Proof. If $z_{0}$ is a global maximum point of $K$, then $z_{0} \in\left\{z_{\delta(1)}, \ldots, z_{\delta(l)}, \beta_{1}, \ldots, \beta_{t}\right\}$.

If $z_{0}=z_{k}$ for some $k \in\{\delta(1), \ldots, \delta(l)\}$, then $g$ can be written in a neighborhood of $z_{k}$ in the form

$$
g=\frac{h}{\left|z-z_{k}\right|^{2-2 \alpha_{k}}}|d z|^{2}, \quad \text { with } h\left(z_{k}\right)>0 .
$$

By (17) and (23), there is a continuous function $H$ on the neighborhood of $z_{k}$ such that

$$
-\frac{1}{3} K^{3}+C K+C^{\prime}=H\left|z-z_{k}\right|^{2 \alpha_{k}}, \quad \text { with } H\left(z_{k}\right) \neq 0 .
$$

Let $z \rightarrow z_{k}$. Then $-\frac{1}{3} K_{1}^{3}+C K_{1}+C^{\prime}=0$, which means $K_{1}$ is one of the roots of $-\frac{1}{3} K^{3}+C K+C^{\prime}=0$.

On the other hand, if $z_{0}=\beta_{m}$ for some $m \in\{1, \ldots, t\}$, then $g=e^{2 \varphi}|d z|^{2}$ and $e^{2 \varphi}$ is a smooth function on a neighborhood of $\beta_{m}$. By (17) and (23), in the neighborhood of $\beta_{m}$, there also exists a continuous function $\widetilde{H}$ such that

$$
-\frac{1}{3} K^{3}+C K+C^{\prime}=\widetilde{H}\left|z-\beta_{m}\right|^{2}, \quad \text { with } \widetilde{H}\left(\beta_{m}\right) \neq 0 .
$$

Let $z \rightarrow \beta_{m}$. Then $-\frac{1}{3} K_{1}^{3}+C K_{1}+C^{\prime}=0$.

Similarly, $K_{2}$ is also one of the roots of $-\frac{1}{3} K^{3}+C K+C^{\prime}=0$.

In fact we have the following finer conclusion about $K_{1}, K_{2},-\left(K_{1}+K_{2}\right)$ and the values of $K$ at the local extremal points.

Proposition 3.2 [Chen et al. 2005]. We have $K_{1}>0$ and $K_{1}>K_{2}>-\left(K_{1}+K_{2}\right)$. The local maxima of $K$ are the same, as are the local minima of $K$.

Now we sketch the proof of necessity in Theorem 1.4, which requires that we determine the values of $c_{k}$ and $c_{m}^{\prime}$ in (24). To do this we need to solve the equation of $K$, that is, (16). Then by its solution, the continuity of $K$, and Equation (1), we can determine $c_{k}$ and $c_{m}^{\prime}$ in (24).

We first solve (16). By Proposition 3.2, every root of $-\frac{1}{3} K^{3}+C K+C^{\prime}=0$ is a simple root. Then we directly suppose

$$
\frac{1}{-\frac{1}{3} K^{3}+C K+C^{\prime}}=\frac{a_{1}}{K-K_{1}}+\frac{a_{2}}{K-K_{2}}+\frac{a_{3}}{K+K_{1}+K_{2}},
$$

by (16) and (24),

$$
\begin{array}{rl}
\left(\frac{a_{1}}{K-K_{1}}+\frac{a_{2}}{K-K_{2}}+\frac{a_{3}}{K+K_{1}+K_{2}}\right) K_{z} & d z \\
& =\left(\sum_{k=j+1}^{n} \frac{c_{k}}{z-z_{k}}+\sum_{m=1}^{s} \frac{c_{m}^{\prime}}{z-\beta_{m}}\right) d z
\end{array}
$$


Dividing (28) by $a_{1}$, we have

$$
\begin{aligned}
\left(\frac{1}{K-K_{1}}+\frac{a-1}{K-K_{2}}+\frac{-a}{K+K_{1}+K_{2}}\right) K_{z} d z= & \\
& \left(\sum_{k=j+1}^{n} \frac{b_{k}}{z-z_{k}}+\sum_{m=1}^{s} \frac{b_{m}^{\prime}}{z-\beta_{m}}\right) d z .
\end{aligned}
$$

We can rewrite (29) as

(30) $\left(\frac{1}{K-K_{1}}+\frac{a-1}{K-K_{2}}+\frac{-a}{K+K_{1}+K_{2}}\right) d K$

$=d\left(\sum_{k=j+1}^{n}\left(b_{k} \log \left(z-z_{k}\right)+\overline{b_{k} \log \left(z-z_{k}\right)}\right)+\sum_{m=1}^{s}\left(b_{m}^{\prime} \log \left(z-\beta_{m}\right)+\overline{b_{m}^{\prime} \log \left(z-\beta_{m}\right)}\right)\right)$

on $\mathbb{C} \backslash\left(\{\right.$ some rays $\left.\} \cup\left\{z_{2}, \ldots, z_{n}, \beta_{1}, \ldots, \beta_{s}\right\}\right)$. Let $b_{k}=d_{k}+\sqrt{-1} e_{k}$ and $b_{m}^{\prime}=$ $d_{m}^{\prime}+\sqrt{-1} e_{m}^{\prime}$, and integrate (30). Then

$$
\begin{aligned}
& \left(K_{1}-K\right)\left(K-K_{2}\right)^{a-1}\left(K+K_{1}+K_{2}\right)^{-a} \\
& \quad=A \prod_{k=j+1}^{n}\left|z-z_{k}\right|^{2 d_{k}} \prod_{k=j+1}^{n} e^{-2 e_{k} \arg \left(z-z_{k}\right)} \prod_{m=1}^{s}\left|z-\beta_{m}\right|^{2 d_{m}^{\prime}} \prod_{m=1}^{s} e^{-2 e_{m}^{\prime} \arg \left(z-\beta_{m}\right)}
\end{aligned}
$$

on the same set as before, where $A$ is a constant. By the continuity of $K$, we have $e_{k}=0$ for $k=j+1, \ldots, n$ and $e_{m}^{\prime}=0$ for $m=1,2, \ldots, s$. Therefore

$$
\left(K_{1}-K\right)\left(K-K_{2}\right)^{a-1}\left(K+K_{1}+K_{2}\right)^{-a}=A \prod_{k=j+1}^{n}\left|z-z_{k}\right|^{2 d_{k}} \prod_{m=1}^{s}\left|z-\beta_{m}\right|^{2 d_{m}^{\prime}}
$$

or

$$
\begin{aligned}
\ln \left(K_{1}-K\right)+(a-1) \ln \left(K-K_{2}\right)+(-a) \ln \left(K+K_{1}+K_{2}\right) \\
=a^{\prime}+2 \sum_{k=j+1}^{n} d_{k} \ln \left|z-z_{k}\right|+2 \sum_{m=1}^{s} d_{m}^{\prime} \ln \left|z-\beta_{m}\right|
\end{aligned}
$$

on $\mathbb{C} \backslash\left\{z_{2}, \ldots, z_{n}, \beta_{1}, \ldots, \beta_{s}\right\}$.

By (28) and (29), $a-1=-\left(2 K_{1}+K_{2}\right) /\left(K_{1}+2 K_{2}\right)$. In [Chen et al. 2005] we obtained

$$
K_{1}=\frac{\pi}{A(g)}\left(2 \alpha_{\max }-\alpha_{\min }\right) \quad \text { and } \quad K_{2}=\frac{\pi}{A(g)}\left(2 \alpha_{\min }-\alpha_{\max }\right),
$$

where $A(g)$ is the area of the HCMU metric, $\alpha_{\max }=\alpha_{\delta(1)}+\cdots+\alpha_{\delta(l)}+t$ is the sum of the angles of the maximum points of $K$, and $\alpha_{\min }=\alpha_{\delta(l+1)}+\cdots+\alpha_{\delta(n-j)}+s-t$ is the sum of the angles of the minimum points of $K$. Since $K_{1}>K_{2}$, by (33) we 
have $\alpha_{\max }>\alpha_{\min }$ and

$$
a-1=-\frac{\alpha_{\delta(1)}+\cdots+\alpha_{\delta(l)}+t}{\alpha_{\delta(l+1)}+\cdots+\alpha_{\delta(n-j)}+s-t}=-\frac{\alpha_{\max }}{\alpha_{\min }} .
$$

Next we compute $d_{k}$ and $d_{m}^{\prime}$. We take a small neighborhood $U_{k}$ of $z_{k}$ for $k \in\{\delta(1), \ldots, \delta(l)\}$. Then $e^{2 \varphi}=h /\left|z-z_{k}\right|^{2-2 \alpha_{k}}$ on $U_{k} \backslash\left\{z_{k}\right\}$, and by (24) we have $1 /|F|^{2}=\hat{h}(z) /\left|z-z_{k}\right|^{2}$, where $\hat{h}(z)$ is a continuous function on $U_{k}$ with $\hat{h}\left(z_{k}\right) \neq 0$. Since $(17)$ holds on $\mathbb{C} \backslash\left\{z_{2}, \ldots, z_{n}, \beta_{1}, \ldots, \beta_{s}\right\}$, we substitute

$$
\begin{aligned}
e^{2 \varphi} & =h /\left|z-z_{k}\right|^{2-2 \alpha_{k}}, \\
1 /|F|^{2} & =\hat{h}(z) /\left|z-z_{k}\right|^{2}, \\
-\frac{1}{3} K^{3}+C K+C^{\prime} & =-\frac{1}{3}\left(K-K_{1}\right)\left(K-K_{2}\right)\left(K+K_{1}+K_{2}\right)
\end{aligned}
$$

into (17) to get

$$
h\left|z-z_{k}\right|^{2 \alpha_{k}} /\left|z-z_{k}\right|^{2}=-\frac{1}{3}\left(K-K_{1}\right)\left(K-K_{2}\right)\left(K+K_{1}+K_{2}\right) \hat{h}(z) /\left|z-z_{k}\right|^{2} .
$$

Thus

$$
\frac{K-K_{1}}{\left|z-z_{k}\right|^{2 \alpha_{k}}}=\frac{-3 h}{\hat{h}} \frac{1}{\left(K-K_{2}\right)\left(K+K_{1}+K_{2}\right)} \quad \text { on } U_{k} \backslash\left\{z_{k}\right\} .
$$

Now if we let $z \rightarrow z_{k}$ here, there is a nonzero constant $T_{k} \in \mathbb{R}$ such that

$$
\lim _{z \rightarrow z_{k}} \frac{K-K_{1}}{\left|z-z_{k}\right|^{2 \alpha_{k}}}=T_{k}
$$

On the other hand, (31) implies that

$$
\left(K_{1}-K\right)\left(K-K_{2}\right)^{a-1}\left(K+K_{1}+K_{2}\right)^{-a}=\left|z-z_{k}\right|^{2 d_{k}} l(z) \quad \text { on } U_{k} \backslash\left\{z_{k}\right\} \text {, }
$$

where $l(z)$ is a continuous function on $U_{k}$ with $l\left(z_{k}\right) \neq 0$. Then

$$
\frac{K_{1}-K}{\left|z-z_{k}\right|^{2 d_{k}}}=l(z)\left(K-K_{2}\right)^{1-a}\left(K+K_{1}+K_{2}\right)^{a} \quad \text { on } U_{k} \backslash\left\{z_{k}\right\} .
$$

Again let $z \rightarrow z_{k}$ here. Then there is also a nonzero constant $A_{k} \in \mathbb{R}$ such that

$$
\lim _{z \rightarrow z_{k}} \frac{K_{1}-K}{\left|z-z_{k}\right|^{2 d_{k}}}=A_{k}
$$

Comparing (34) with (36) we know $d_{k}=\alpha_{k}$ for $k=\delta(1), \ldots, \delta(l)$. Similarly $d_{k^{\prime}}=(a-1) \alpha_{k^{\prime}}, k^{\prime}=\delta(l+1), \ldots, \delta(n-j)$ and $d_{m}^{\prime}=1,1 \leq m \leq t, d_{m^{\prime}}^{\prime}=$ $a-1, t+1 \leq m^{\prime} \leq s$. Finally by (24), (28) and (29) we obtain (11). Thus we have proved the necessity part of Theorem 1.4. 
3.2. Proof of sufficiency. If (11) holds, we choose two real numbers $K_{1}$ and $K_{2}$ such that $K_{1}>0$ and

$$
K_{2} / K_{1}=\left(2 \alpha_{\min }-\alpha_{\max }\right) /\left(2 \alpha_{\max }-\alpha_{\min }\right) .
$$

Let

$$
\begin{aligned}
\frac{1}{\sigma F} & =\sum_{k=1}^{l} \frac{\alpha_{\delta(k)}}{z-z_{\delta(k)}}+\sum_{k^{\prime}=l+1}^{n-j} \frac{(a-1) \alpha_{\delta\left(k^{\prime}\right)}}{z-z_{\delta\left(k^{\prime}\right)}}+\sum_{m=1}^{t} \frac{1}{z-\beta_{m}}+\sum_{m^{\prime}=t+1}^{s} \frac{a-1}{z-\beta_{m^{\prime}}} \\
& =\frac{B \prod_{i^{\prime}=2}^{j}\left(z-z_{i^{\prime}}\right)^{\alpha_{i^{\prime}}-1}}{\prod_{k=j+1}^{n}\left(z-z_{k}\right) \prod_{m=1}^{s}\left(z-\beta_{m}\right)},
\end{aligned}
$$

where $1 / \sigma=\left(2 K_{1}+K_{2}\right)\left(K_{2}-K_{1}\right) / 3$.

Lemma 3.1. There is a unique real solution of the system

$$
\left\{\begin{aligned}
K_{z} & =-\frac{1}{3}\left(K-K_{1}\right)\left(K-K_{2}\right)\left(K+K_{1}+K_{2}\right) / F, \\
K\left(z_{0}\right) & =K_{0} \text { for } z_{0} \in \mathbb{C} \backslash\left\{\beta_{1}, \ldots, \beta_{s}, z_{j+1}, \ldots, z_{n}\right\}, K_{2}<K_{0}<K_{1} .
\end{aligned}\right.
$$

Proof. We can directly integrate (38) in $\left(K_{2}, K_{1}\right) \times\left(\mathbb{C} \backslash\left\{\beta_{1}, \ldots, \beta_{s}, z_{j+1}, \ldots, z_{n}\right\}\right)$ and get

$$
\begin{aligned}
\ln \left(K_{1}-K\right)+(a-1) \ln \left(K-K_{2}\right)+(-a) \ln \left(K+K_{1}+K_{2}\right) & \\
=a^{\prime}+2 \sum_{k=1}^{l} \alpha_{\delta(k)} \ln \mid z & -z_{\delta(k)}\left|+2 \sum_{k^{\prime}=l+1}^{n}(a-1) \alpha_{\delta\left(k^{\prime}\right)} \ln \right| z-z_{\delta\left(k^{\prime}\right)} \mid \\
& +2 \sum_{m=1}^{t} \ln \left|z-\beta_{m}\right|+2 \sum_{m^{\prime}=t+1}^{s}(a-1) \ln \left|z-\beta_{m^{\prime}}\right|
\end{aligned}
$$

For simplicity we write this equation in the form $G(K, z, \bar{z})=a^{\prime}$, which makes it clear that the equation uniquely determines a surface in the set $\left(K_{2}, K_{1}\right) \times$ $\left(\mathbb{C} \backslash\left\{\beta_{1}, \ldots, \beta_{s}, z_{j+1}, \ldots, z_{n}\right\}\right)$. However, $\partial G / \partial K \neq 0$ in this set, so we get a graph $K=K(z, \bar{z})$ on $\mathbb{C} \backslash\left\{\beta_{1}, \ldots, \beta_{s}, z_{j+1}, \ldots, z_{n}\right\}$. Clearly it satisfies (38).

Since $K$ satisfies (38), it is smooth on its domain and satisfies (31). Then

$\lim _{z \rightarrow z_{\delta(k)}} K=K_{1} \quad$ for $k=1, \ldots, l, \quad \lim _{z \rightarrow z_{\delta\left(k^{\prime}\right)}} K=K_{2} \quad$ for $k^{\prime}=l+1, \ldots, n-j$,

$\lim _{z \rightarrow \beta_{m}} K=K_{1} \quad$ for $m=1, \ldots, t, \quad \lim _{z \rightarrow \beta_{m^{\prime}}} K=K_{2}, \quad$ for $m^{\prime}=t+1, \ldots, s ;$

and there exists a real number $K_{\infty}$ with $K_{2}<K_{\infty}<K_{1}$ such that $\lim _{z \rightarrow \infty} K=K_{\infty}$. Therefore $K$ is a continuous function on $S^{2}$.

Now define

$$
e^{2 \varphi}=\frac{-\frac{1}{3}\left(K-K_{1}\right)\left(K-K_{2}\right)\left(K+K_{1}+K_{2}\right)}{|F|^{2}} \quad \text { on } \mathbb{C} \backslash\left\{\beta_{1}, \ldots, \beta_{s}, z_{2}, \ldots, z_{n}\right\}
$$


and $g=e^{2 \varphi}|d z|^{2}$. Then $e^{2 \varphi}>0$ and is smooth on $\mathbb{C} \backslash\left\{\beta_{1}, \ldots, \beta_{s}, z_{2}, \ldots, z_{n}\right\}$. Let

$$
-\frac{1}{3}\left(K-K_{1}\right)\left(K-K_{2}\right)\left(K+K_{1}+K_{2}\right)=-\frac{1}{3} K^{3}+C K+C^{\prime} .
$$

Then a direct calculation shows

$$
\begin{aligned}
\Delta K & =\left(-K^{2}+C\right) e^{2 \varphi}, \\
-\Delta \varphi & =K e^{2 \varphi}, \\
F & =K_{\bar{z}} e^{-2 \varphi} .
\end{aligned}
$$

These imply that $g$ is an HCMU metric on $\mathbb{C} \backslash\left\{\beta_{1}, \ldots, \beta_{s}, z_{2}, \ldots, z_{n}\right\}$.

Next we prove $e^{2 \varphi}$ is actually smooth at $\beta_{m}$ for $m=1, \ldots, s$. For a fixed $m$ with $m=1,2, \ldots, t$, let $\Omega_{m}$ be a neighborhood of $\beta_{m}$. Then by (40) we have on $\Omega_{m} \backslash\left\{\beta_{m}\right\}$ that

$$
e^{2 \varphi}=\frac{K_{1}-K}{\left|z-\beta_{m}\right|^{2}}\left(K-K_{2}\right)\left(K+K_{1}+K_{2}\right) G_{1}(z)
$$

and by (31)

$$
\frac{K_{1}-K}{\left|z-\beta_{m}\right|^{2}}=\left(K-K_{2}\right)^{1-a}\left(K+K_{1}+K_{2}\right)^{a} G_{2}(z)
$$

so

$$
e^{2 \varphi}=\left(K-K_{2}\right)^{2-a}\left(K+K_{1}+K_{2}\right)^{1+a} G_{3}(z),
$$

where $G_{1}(z), G_{2}(z)$ and $G_{3}(z)$ are smooth nonvanishing functions on $\Omega_{m}$. Then $e^{2 \varphi}$ is a continuous function and $e^{2 \varphi}>0$ on $\Omega_{m}$. However, (43) holds on $\Omega_{m} \backslash\left\{\beta_{m}\right\}$, which implies that the first weak derivatives of $K$ exist on $\Omega_{m}$. Furthermore, by (41), we have $\Delta K \in L^{q}\left(\Omega_{m}\right)$, where $q$ is big enough. Then by the CalderonZygmund inequality, $K \in W^{2, q}\left(U_{m}\right)$ for $U_{m} \subset \subset \Omega_{m}$, and $K \in C^{1}\left(U_{m}\right)$ by the Sobolev embedding theorem. Again by (43) we get using a bootstrap technique that $K \in C^{\infty}\left(U_{m}\right)$. Thus, $e^{2 \varphi}$ is smooth at $\beta_{m}$ by (44). Similarly $e^{2 \varphi}$ is smooth at $\beta_{m^{\prime}}$ for $m^{\prime}=t+1, \ldots, s$. Consequently on $\mathbb{C} \backslash\left\{z_{2}, \ldots, z_{n}\right\}$, the function $e^{2 \varphi}$ is smooth, positive and satisfies (41), (42) and (43).

Furthermore, by (31) and (40) one can check that $g$ satisfies the angle condition at $z_{i}$ for $i=2, \ldots, n$. In a neighborhood of $p_{1}=\infty$, let $w=1 / z$. Then $g$ can be written as $\left(\tilde{h} /|w|^{2-2 \alpha_{1}}\right)|d w|^{2}$, where $\tilde{h}>0$ is a continuous function that is smooth except at the origin.

Now we prove that the area and the energy of the metric are both finite. Since $K$ is bounded, we only need prove the area is finite. Because

$$
A(g)=\frac{\sqrt{-1}}{2} \int_{\mathbb{C} \backslash\left\{z_{2}, \ldots, z_{n}, \beta_{1}, \ldots, \beta_{s}\right\}} e^{2 \varphi} d z \wedge d \bar{z}
$$


by (43), we have

$$
\begin{aligned}
& A(g)= \frac{\sqrt{-1}}{2} \int \frac{K_{\bar{z}}}{F} d z \wedge d \bar{z} \\
&=\frac{\sigma \sqrt{-1}}{2} \int K_{\bar{z}}\left(\sum_{k=1}^{l} \frac{\alpha_{\delta(k)}}{z-z \delta(k)}+\sum_{k^{\prime}=l+1}^{n-j} \frac{(a-1) \alpha_{\delta\left(k^{\prime}\right)}}{z-z_{\delta\left(k^{\prime}\right)}}\right. \\
&\left.\quad+\sum_{m=1}^{t} \frac{1}{z-\beta_{m}}+\sum_{m^{\prime}=t+1}^{s} \frac{a-1}{z-\beta_{m^{\prime}}}\right) d z \wedge d \bar{z},
\end{aligned}
$$

where both integrals are over $\mathbb{C} \backslash\left\{z_{2}, \ldots, z_{n}, \beta_{1}, \ldots, \beta_{s}\right\}$. Let $z=z_{\delta(k)}+r e^{\sqrt{-1} \theta}$. Then we may compute $k$-summand in the first term of the right side of (46) by

$$
\begin{aligned}
& \frac{\sqrt{-1}}{2} \int \frac{K_{\bar{z}}}{z-z_{\delta(k)}} d z \wedge d \bar{z} \\
& \quad=\frac{1}{2} \int_{0}^{\infty} \int_{0}^{2 \pi}\left(\frac{\partial K}{\partial r}+\sqrt{-1} \frac{1}{r} \frac{\partial K}{\partial \theta}\right) d r d \theta=\pi\left(K(\infty)-K_{1}\right) .
\end{aligned}
$$

The other terms can be computed similarly. Therefore

$$
\begin{aligned}
A(g)= & \sigma \pi\left(\sum_{k=1}^{l} \alpha_{\delta(k)}\left(K(\infty)-K_{1}\right)+(a-1) \sum_{k^{\prime}=l+1}^{n-j} \alpha_{\delta\left(k^{\prime}\right)}\left(K(\infty)-K_{2}\right)\right. \\
& \left.+\sum_{m=1}^{t}\left(K(\infty)-K_{1}\right)+(a-1) \sum_{m^{\prime}=t+1}^{s}\left(K(\infty)-K_{2}\right)\right) \\
= & \frac{\pi\left(2 \alpha_{\max }-\alpha_{\min }\right)}{K_{1}}
\end{aligned}
$$

Thus we see that $g$ is an HCMU metric on $S^{2}$ with finite area and energy. The points $z_{1}, \ldots, z_{j}$, the points $z_{\delta(1)}, \ldots, z_{\delta(l)}, \beta_{1}, \ldots, \beta_{t}$ and the points $z_{\delta(l+1)}, \ldots, z_{\delta(n-j)}$, $\beta_{t+1}, \ldots, \beta_{s}$ are respectively the saddle, maximum, and minimum points of $K$. This completes the proof of sufficiency.

As an application of Theorem 1.4, we obtain the following nonexistence result.

Example 3.1. Let $S^{2}=\mathbb{C} \cup\{\infty\}$ with $p_{1}=\infty, p_{2}=0, p_{3}=1$ and $p_{4}=2$. Then there is no HCMU metric on $S^{2}$ with the angle $2 \pi \cdot 3$ at $p_{1}$, the angle $2 \pi \cdot \frac{1}{2}$ at $p_{2}$, the angle $2 \pi \cdot \frac{1}{2}$ at $p_{3}$, and the angle $2 \pi \cdot \frac{1}{2}$ at $p_{4}$.

Proof. Suppose there is an HCMU metric on $S^{2}$ satisfying the condition above. Then by (10) there exist saddle points of $K$. Since the angles at the saddle points of $K$ must be integers, $p_{1}=\infty$ is the unique saddle point of $K$. Also $s=1$ by (10); that is, there is only one smooth critical point of $K$. Thus there are eight cases for the points $p_{2}, p_{3}, p_{4}$ depending on whether these points are maxima or minima 
for $K$. Of course, all three cannot be minima; otherwise the smooth critical point of $K$ must be the maximum point of $K$, which implies $\alpha_{\max }=1$ and $\alpha_{\min }=\frac{3}{2}$, so $\alpha_{\max }<\alpha_{\min }$, an impossibility. To exclude other cases we need to prove in each of them there is no solution of the Equation (11). For instance, we will consider the case that $p_{2}, p_{3}$ and $p_{4}$ are respectively maximum, minimum and minimum points of $K$. In this case, the smooth critical point $\beta$ of $K$ must be the maximum point of $K$, and $a-1=-\left(\frac{1}{2}+1\right) /\left(\frac{1}{2}+\frac{1}{2}\right)=-\frac{3}{2}$. Hence by (11) we have

$$
\frac{\frac{1}{2}}{z}+\frac{\frac{1}{2}\left(-\frac{3}{2}\right)}{z-1}+\frac{\frac{1}{2}\left(-\frac{3}{2}\right)}{z-2}+\frac{1}{z-\beta}=\frac{B}{\cdots} .
$$

Because (49) is an identity we get the two equations

$$
\begin{aligned}
\frac{1}{2}(-1-2-\beta)-\frac{3}{4}(-2-\beta)-\frac{3}{4}(-1-\beta)-1-2 & =0, \\
\frac{1}{2}(2+\beta+2 \beta)-\frac{3}{4} 2 \beta-\frac{3}{4} \beta+2 & =0 .
\end{aligned}
$$

From the first equation we get $\beta=\frac{9}{4}$, but from the second we get $\beta=4$, a contradiction. Therefore there is no solution of (11) in this case. All other possibilities may be handled similarly.

From the proof of the necessity of Theorem 1.4 we get an explicit construction of an HCMU metric on $S^{2}$ :

Theorem 3.1. Let $S^{2}=\mathbb{C} \cup\{\infty\}$ with $p_{1}=\infty$ and $p_{2}=z_{2}, \ldots, p_{n}=z_{n}$. Then $\alpha_{1}, \ldots, \alpha_{n}$ are positive real numbers, and $\alpha_{1}, \ldots, \alpha_{j}$ for $j>0$ are integers with $\alpha_{i^{\prime}} \geq 2$ for $i^{\prime}=1, \ldots, j$. If there exists an HCMU metric on $S^{2}$ such that $1, \ldots, z_{j}$ are the saddle points of $K$, and $z_{j+1}, \ldots, z_{j+l}$ and $z_{j+l+1}, \ldots, z_{n}$ are respectively the maximum and minimum points of $K$, then the HCMU metric can be written in the form

$$
g=e^{2 \varphi}|d z|^{2} \quad \text { on } \mathbb{C} \backslash\left\{z_{2}, \ldots, z_{n}\right\}
$$

with

$$
e^{2 \varphi}=-\frac{1}{3}\left(K-K_{1}\right)\left(K-K_{2}\right)\left(K+K_{1}+K_{2}\right) /|F|^{2}
$$

and $K$ is determined by the equation

$$
\begin{aligned}
& \left(K_{1}-K\right)\left(K-K_{2}\right)^{a-1}\left(K+K_{1}+K_{2}\right)^{-a}= \\
& A \prod_{k=j+1}^{j+l}\left|z-z_{k}\right|^{2 \alpha_{k}} \prod_{k^{\prime}=j+l+1}^{n}\left|z-z_{k^{\prime}}\right|^{2(a-1) \alpha_{k^{\prime}}} \prod_{m=1}^{t}\left|z-\beta_{m}\right|^{2} \prod_{m^{\prime}=t+1}^{s}\left|z-\beta_{m^{\prime}}\right|^{2(a-1)} .
\end{aligned}
$$


Meanwhile $1 / F$ has the expression

$$
\begin{aligned}
\frac{1}{F} & =\frac{B \prod_{i^{\prime}=2}^{j}\left(z-z_{i^{\prime}}\right)^{\alpha_{i^{\prime}}-1}}{\prod_{k=j+1}^{n}\left(z-z_{k}\right) \prod_{m=1}^{s}\left(z-\beta_{m}\right)} \\
& =\sigma\left(\sum_{k=j+1}^{j+l} \frac{\alpha_{k}}{z-z_{k}}+\sum_{k^{\prime}=j+l+1}^{n} \frac{(a-1) \alpha_{k^{\prime}}}{z-z_{k^{\prime}}}+\sum_{m=1}^{t} \frac{1}{z-\beta_{m}}+\sum_{m^{\prime}=t+1}^{s} \frac{a-1}{z-\beta_{m^{\prime}}}\right) .
\end{aligned}
$$

Here $K_{1}$ is the maximum of $K$ that is positive, $K_{2}$ is the minimum of $K$ and

$$
\frac{K_{2}}{K_{1}}=\frac{2 \alpha_{\min }-\alpha_{\max }}{2 \alpha_{\max }-\alpha_{\min }}
$$

where $\alpha_{\max }=\alpha_{j+1}+\cdots+\alpha_{j+l}+t, \quad \alpha_{\min }=\alpha_{j+l+1}+\cdots+\alpha_{n}+s-t$ and $a-1=-\alpha_{\max } / \alpha_{\min }$. Also $A$ and $B$ are constants, $1 / \sigma=\left(K_{2}-K_{1}\right)\left(2 K_{1}+K_{2}\right) / 3$; $s=2+\sum_{i^{\prime}=1}^{j} \alpha_{i^{\prime}}-n$ is the number of the smooth extremal points of $K$; and $\beta_{1}, \ldots, \beta_{t}$ and $\beta_{t+1}, \ldots, \beta_{s}$ are respectively the smooth maximum and minimum points of $K$.

\section{Proof of Theorem 1.5}

4.1. Proof of necessity. Let $\pi: \mathbb{C} \longrightarrow \mathbb{C} / \Gamma$ be the canonical map. In one elementary parallelogram $P_{\mathrm{o}}$,

$$
\nabla K=\sqrt{-1} F(z) \frac{\partial}{\partial z} .
$$

One can check that $F(z)$ can be extended to a meromorphic function $\widetilde{F}$ on $T^{2}$. Then $\widetilde{F} \circ \pi$ is an elliptic function on $\mathbb{C}$. On $P_{\mathrm{o}}$ we assume $p_{i}=z_{i}$ for $i=1,2, \ldots, n$ and $\beta_{1}, \ldots, \beta_{s}$ are the smooth extremal points of $K$. Then by Proposition 2.2, $z_{1}, \ldots, z_{j}$ are the poles of $\widetilde{F}$ and $z_{j+1}, \ldots, z_{n}, \beta_{1}, \ldots, \beta_{s}$ are the zeros of $\widetilde{F}$. By the properties of elliptic functions [Lang 1987], there exist $n_{1}, n_{2} \in \mathbb{Z}$ such that $\sum_{i^{\prime}=1}^{j}\left(\alpha_{i^{\prime}}-1\right) z_{i^{\prime}}-\sum_{k=j+1}^{n} z_{k}-\sum_{m=1}^{s} \beta_{m}=n_{1} w_{1}+n_{2} w_{2}$, which is (13). Also there exist $B, c_{0}, c_{j+1}, \ldots, c_{n}$ and $c_{1}^{\prime}, \ldots, c_{s}^{\prime} \in \mathbb{C}$ such that

$$
\frac{1}{\widetilde{F} \circ \pi}=\frac{B \prod_{i^{\prime}=1}^{j} \sigma\left(z-z_{i^{\prime}}\right)^{\left(\alpha_{i^{\prime}}-1\right)}}{\prod_{k=j+1}^{n} \sigma\left(z-z_{k}\right) \prod_{m=1}^{s-1} \sigma\left(z-\beta_{m}\right) \sigma\left(z-\beta_{s}-n_{1} w_{1}-n_{2} w_{2}\right)}
$$

or

$$
\frac{1}{\widetilde{F} \circ \pi}=c_{0}+\sum_{k=j+1}^{n} c_{k} \zeta\left(z-z_{k}\right)+\sum_{m=1}^{s} c_{m}^{\prime} \zeta\left(z-\beta_{m}\right),
$$

where $\sum_{k=j+1}^{n} c_{k}+\sum_{m=1}^{s} c_{m}^{\prime}=0$. Proceeding like the $S^{2}$ case, we also suppose there are a permutation $\delta$ of $j+1, \ldots, n$, an integer $l$ with $0 \leq l \leq n-j$, and an integer $t$ in $0 \leq t \leq s$, such that $z_{\delta(1)}, \ldots, z_{\delta(l)}, \beta_{1}, \ldots, \beta_{t}$ and $z_{\delta(l+1)}, \ldots, z_{\delta(n-j)}$, $\beta_{t+1}, \ldots, \beta_{s}$ are respectively the maximum points and minimum points of $K$. 
On the other hand, we define $\widetilde{K}=K \circ \pi$. Then $\widetilde{K}(z+w)=\widetilde{K}(z)$, where $w=n w_{1}+m w_{2}$ for $n, m \in \mathbb{Z}$. Also, by (16)

$$
\widetilde{K}_{z}=\frac{-\frac{1}{3} \widetilde{K}^{3}+C \widetilde{K}+C^{\prime}}{\widetilde{F} \circ \pi} \text { on } \mathbb{C} \backslash\left[z_{1}, z_{2}, \ldots, z_{n}, \beta_{1}, \ldots, \beta_{s}\right],
$$

where $\left[z_{1}, \ldots, z_{n}, \beta_{1}, \ldots, \beta_{s}\right]=\bigcup_{w}\left(\left\{z_{1}, \ldots, z_{n}, \beta_{1}, \ldots, \beta_{s}\right\}+w\right)$. Then, like the $S^{2}$ case, we have

$$
-\frac{1}{3} \widetilde{K}^{3}+C \widetilde{K}+C^{\prime}=-\frac{1}{3}\left(\widetilde{K}-K_{1}\right)\left(\widetilde{K}-K_{2}\right)\left(\widetilde{K}+K_{1}+K_{2}\right),
$$

where $K_{1}=K_{\max }$ and $K_{2}=K_{\min }$. Then we have

$$
\begin{aligned}
&\left(\frac{1}{\widetilde{K}-K_{1}}+\frac{a-1}{\widetilde{K}-K_{2}}+\frac{-a}{\widetilde{K}+K_{1}+K_{2}}\right) \widetilde{K}_{z}= \\
& \lambda+\sum_{k=j+1}^{n} d_{k} \zeta\left(z-z_{k}\right)+\sum_{m=1}^{s} d_{m}^{\prime} \zeta\left(z-\beta_{m}\right) .
\end{aligned}
$$

Lemma 4.1. Both $d_{k}$ and $d_{m}^{\prime}$ are real numbers.

Proof. Pick an elementary parallelogram $P_{z}$ such that there are neither zeros nor poles of $\widetilde{F} \circ \pi$ on $\partial P_{z}$. We denote the poles of $1 /(\widetilde{F} \circ \pi)$ in $P_{z}$ by $q_{b}$ for $b=1,2, \ldots, n-j+s$. For simplicity we denote $\sigma\left(z-q_{b}\right)$ by $f_{b}$.

Since $\sigma^{\prime}(0) \neq 0$, for a given $b$ there is a disk $D_{b} \ni q_{b}$ such that $f_{b}$ is biholomorphic on $D_{b}$. Let $L_{\tilde{b}}=\left\{t f_{b}\left(q_{\tilde{b}}\right) \mid t \geq 0\right\}$ for any $\tilde{b} \neq b$. Then $\bigcap_{\tilde{b} \neq b} f_{b}^{-1}\left(L_{\tilde{b}}\right)^{c} \cap D_{b}$ is nonempty (if it were empty, then $f_{b}\left(D_{b}\right) \subset \bigcup_{\tilde{b} \neq b} L_{\tilde{b}}$, which contradicts the fact that $f_{b}\left(D_{b}\right)$ is open). Now take a $Z_{b}$ in this intersection, and let $S_{b}=\left\{t f_{b}\left(Z_{b}\right) \mid t \geq 0\right\}$. Then $q_{\tilde{b}} \in f_{b}^{-1}\left(S_{b}\right)^{c}$ for all $\tilde{b} \neq b$.

Now we fix a $b \in\{1,2, \ldots, n-j+s\}$. For any $\tilde{b} \neq b$ there is a $Z_{\tilde{b}} \in D_{\tilde{b}}$ given as above. Then $q_{b} \in \bigcap_{\tilde{b} \neq b} f_{\tilde{b}}^{-1}\left(S_{\tilde{b}}\right)^{c}$. Since $f_{\tilde{b}}^{-1}\left(S_{\tilde{b}}\right)^{c}$ is open, there exists a disk $D_{b}^{\prime} \subset \bigcap_{\tilde{b} \neq b} f_{\tilde{b}}^{-1}\left(S_{\tilde{b}}\right)^{c} \cap D_{b}$ such that $q_{b} \in D_{b}^{\prime}$.

By the formula $\zeta(z)=\sigma^{\prime}(z) / \sigma(z)$, we may rewrite (57) as

$$
\left(\frac{1}{\widetilde{K}-K_{1}}+\frac{a-1}{\widetilde{K}-K_{2}}+\frac{-a}{\widetilde{K}+K_{1}+K_{2}}\right) \widetilde{K}_{z}=\lambda+d_{b} f_{b}^{\prime} / f_{b}+\sum_{\tilde{b} \neq b} d_{\tilde{b}} f_{\tilde{b}}^{\prime} / f_{\tilde{b}}
$$

on $P_{z} \backslash\left\{q_{1}, \ldots, q_{n-j+s}\right\}$. For any $\tilde{b} \neq b$, regard $\mathbb{C} \backslash S_{\tilde{b}}$ as the simple-valued domain of the logarithm defined in the image space of $f_{\tilde{b}}$. Then $f_{\tilde{b}}^{\prime} / f_{\tilde{b}}=\left(\log f_{\tilde{b}}\right)^{\prime}$ on $f_{\tilde{b}}^{-1}\left(S_{\tilde{b}}\right)^{c}$. It follows that $f_{\tilde{b}}^{\prime} / f_{\tilde{b}}=\left(\log f_{\tilde{b}}\right)^{\prime}$ on $D_{b}^{\prime}$ for all $\tilde{b} \neq b$. Since $f_{b}$ is biholomorphic on $D_{b}$, there is a domain $V_{b}$ with $q_{b} \in V_{b} \subset D_{b}^{\prime}$ such that $f_{b}\left(V_{b}\right)$ is a disk $\widetilde{D}$ centered at the origin. We denote the inverse map of $f_{b}$ restricted to $V_{b}$ by $g_{b}$. Suppose $t_{0} f_{b}\left(Z_{b}\right) \in \partial \widetilde{D}$. Then $g_{b}$ maps the domain $\widetilde{D} \backslash S_{b}$ onto $\widetilde{V}_{b}:=V_{b} \backslash\left\{g_{b}\left(t f_{b}\left(Z_{b}\right)\right)\right.$ for $\left.0 \leq t<t_{0}\right\}$. So $\widetilde{V}_{b} \subset f_{b}^{-1}\left(S_{b}\right)^{c}$. Regard $\mathbb{C} \backslash S_{b}$ as 


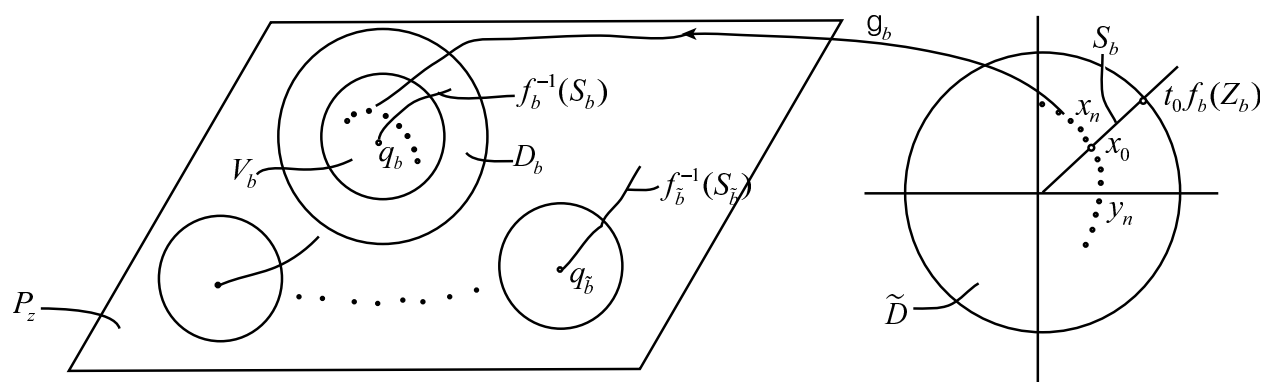

the simple-valued domain of the logarithm defined in the image space of $f_{b}$. Then $f_{b}^{\prime} / f_{b}=\left(\log f_{b}\right)^{\prime}$ on $\widetilde{V}_{b}$ since $\widetilde{V}_{b} \subset f_{b}^{-1}\left(S_{b}\right)^{c}$. We integrate (58) and obtain

$$
\begin{aligned}
& d\left(\ln \left(K_{1}-\widetilde{K}\right)+(a-1) \ln \left(\widetilde{K}-K_{2}\right)+(-a) \ln \left(\widetilde{K}+K_{1}+K_{2}\right)\right) \\
& \quad=d(\lambda z+\bar{\lambda} \bar{z})+d\left(d_{b} \log f_{b}+\overline{d_{b} \log f_{b}}\right)+\sum_{\tilde{b} \neq b} d\left(d_{\tilde{b}} \log f_{\tilde{b}}+\overline{d_{\tilde{b}} \log f_{\tilde{b}}}\right)
\end{aligned}
$$

on $\widetilde{V}_{b}$. Let $d_{b}=d_{1 b}+\sqrt{-1} d_{2 b}$ and $d_{\tilde{b}}=d_{1 \tilde{b}}+\sqrt{-1} d_{2 \tilde{b}}$ for $\tilde{b} \neq b$. Then (59) can be written in the form

$$
\begin{aligned}
\left(K_{1}-\widetilde{K}\right)\left(\widetilde{K}-K_{2}\right)^{a-1}\left(\widetilde{K}+K_{1}+K_{2}\right)^{-a} & \\
= & A e^{\lambda z+\bar{\lambda} \bar{z}}\left|f_{b}\right|^{2 d_{1 b}} e^{-2 d_{2 b} \arg f_{b}} \prod_{\tilde{b} \neq b}\left|f_{\tilde{b}}\right|^{2 d_{1 \tilde{b}}} e^{-2 d_{2 \tilde{b}} \arg f_{\tilde{b}}}
\end{aligned}
$$

In $\widetilde{D}$ we take $\left\{x_{n}\right\}$ and $\left\{y_{n}\right\}$ to be two sequences on opposite sides of $S_{b}$, and let $x_{n} \rightarrow x_{0}$ and $y_{n} \rightarrow x_{0}$, where $x_{0} \in \widetilde{D} \cap S_{b}$. See the figure above.

Now we substitute $\left\{g_{b}\left(x_{n}\right)\right\}$ and $\left\{g_{b}\left(y_{n}\right)\right\}$ into (60) and take limits. Since $V_{b} \subset$ $f_{\tilde{b}}^{-1}\left(S_{\tilde{b}}\right)^{c}$ for all $\tilde{b} \neq b, \arg f_{\tilde{b}}$ is continuous on $V_{b}$. Then if we substitute $\left\{g_{b}\left(x_{n}\right)\right\}$ and $\left\{g_{b}\left(y_{n}\right)\right\}$ into any term in (60) except for $e^{-2 d_{2 b} \text { arg } f_{b}}$ and take limits, the limits are the same. However, if we substitute $\left\{g_{b}\left(x_{n}\right)\right\}$ and $\left\{g_{b}\left(y_{n}\right)\right\}$ into $e^{-2 d_{2 b} \text { arg } f_{b}}$ and take limits, there is a difference of $e^{-2 d_{2 b} 2 \pi}$ between the limits. Thus $d_{2 b}=0$, completing the proof of the lemma.

Now there exists an $A \in \mathbb{R}$ on $P_{\mathrm{o}} \backslash\left\{z_{1}, z_{2}, \ldots, z_{n}, \beta_{1}, \ldots, \beta_{s}\right\}$ such that

$$
\begin{aligned}
& \ln \left(K_{1}-\widetilde{K}\right)+(a-1) \ln \left(\widetilde{K}-K_{2}\right)-a \ln \left(\widetilde{K}+K_{1}+K_{2}\right)= \\
& A+\lambda z+\bar{\lambda} \bar{z}+2 \sum_{k=j+1}^{n} d_{k} \ln \left|\sigma\left(z-z_{k}\right)\right|+2 \sum_{m=1}^{s} d_{m}^{\prime} \ln \left|\sigma\left(z-\beta_{m}\right)\right| .
\end{aligned}
$$

By the periodicity of $\widetilde{K}$ we get

$$
\begin{aligned}
\lambda w_{1}+\bar{\lambda} \bar{w}_{1}=2 \sum_{k=j+1}^{n} d_{k}\left(\ln \left|\sigma\left(z_{0}-z_{k}\right)\right|-\ln \left|\sigma\left(z_{0}+w_{1}-z_{k}\right)\right|\right) & \\
& +2 \sum_{m=1}^{s} d_{m}^{\prime}\left(\ln \left|\sigma\left(z_{0}-\beta_{m}\right)\right|-\ln \left|\sigma\left(z_{0}+w_{1}-\beta_{m}\right)\right|\right),
\end{aligned}
$$


where $z_{0} \in \partial P_{\mathrm{o}} \backslash\left\{z_{1}, \ldots, z_{n}, \beta_{1}, \ldots, \beta_{s}\right\}$. Since $\sigma\left(z+w_{1}\right)=-\sigma(z) e^{\eta_{1}\left(z+w_{1} / 2\right)}$, where $\eta_{1}=2 \zeta\left(w_{1} / 2\right)$, Equation (62) becomes

$$
\begin{aligned}
\lambda w_{1}+\bar{\lambda} \bar{w}_{1} & =-2 \sum_{k=j+1}^{n} d_{k} \operatorname{Re}\left(\eta_{1}\left(z_{0}-z_{k}+\frac{1}{2} w_{1}\right)\right)-2 \sum_{m=1}^{s} d_{m}^{\prime} \operatorname{Re}\left(\eta_{1}\left(z_{0}-\beta_{m}+\frac{1}{2} w_{1}\right)\right) \\
& =\operatorname{Re}\left(\eta_{1} 2\left(\sum_{k=j+1}^{n} d_{k} z_{k}+\sum_{m=1}^{s} d_{m}^{\prime} \beta_{m}\right)\right)=\operatorname{Re}\left(\eta_{1} \rho\right),
\end{aligned}
$$

where $\rho=2\left(\sum_{k=j+1}^{n} d_{k} z_{k}+\sum_{m=1}^{s} d_{m}^{\prime} \beta_{m}\right)$. Similarly $\lambda w_{2}+\bar{\lambda} \bar{w}_{2}=\operatorname{Re}\left(\eta_{2} \rho\right)$, where $\eta_{2}=2 \zeta\left(w_{2} / 2\right)$. Then we obtain

$$
\lambda=\frac{1}{w_{1} \bar{w}_{2}-w_{2} \bar{w}_{1}}\left(\operatorname{Re}\left(\eta_{1} \rho\right) \bar{w}_{2}-\operatorname{Re}\left(\eta_{2} \rho\right) \bar{w}_{1}\right) .
$$

Furthermore, $\widetilde{K}$ satisfies (61) on $\mathbb{C} \backslash\left[z_{1}, \ldots, z_{n}, \beta_{1}, \ldots, \beta_{s}\right]$. By an argument similar to the one in the $S^{2}$ case,

$$
\begin{aligned}
d_{k} & = \begin{cases}\alpha_{k} & \text { for } k=\delta(1), \ldots, \delta(l), \\
(a-1) \alpha_{k} & \text { for } k=\delta(l+1), \ldots, \delta(n-j),\end{cases} \\
d_{m}^{\prime} & = \begin{cases}1 & \text { for } 1 \leq m \leq t, \\
a-1 & \text { for } t+1 \leq m \leq s,\end{cases} \\
a-1 & =-\frac{\alpha_{\delta(1)}+\cdots+\alpha_{\delta(l)}+t}{\alpha_{\delta(l+1)}+\cdots+\alpha_{\delta(n-j)}+s-t} .
\end{aligned}
$$

Then by (53), (54), (55) and (57) we get (14). This completes the proof of necessity.

4.2. Proof of sufficiency. In fact one can follow the proof of the sufficiency for Theorem 1.4 to prove the sufficiency for Theorem 1.5. The only thing left is to calculate the area of an HCMU metric on $T^{2}$. Take an elementary parallelogram $P$ and suppose on $\partial P$ there is no singular point of the meromorphic function

$$
\frac{1}{\sigma F}=\lambda+\sum_{k=j+1}^{n} d_{k} \zeta\left(z-z_{k}\right)+\sum_{m=1}^{s} d_{m}^{\prime} \zeta\left(z-\beta_{m}\right),
$$

where we use the notations of (64) to denote $d_{k}$ and $d_{m}^{\prime}$. The area is

$$
A(g)=\lim _{\varepsilon \rightarrow 0} \frac{\sqrt{-1}}{2} \int_{P \backslash\left(\bigcup_{k} D_{\varepsilon}\left(z_{k}\right) \cup \bigcup_{m} D_{\varepsilon}\left(\beta_{m}\right)\right)} \frac{K_{\bar{z}}}{F} d z \wedge d \bar{z},
$$


where $D_{\varepsilon}(z)$ is a round disk centered at $z$ with the radius $\varepsilon$. By Stokes's theorem,

$$
\begin{aligned}
& \frac{\sqrt{-1}}{2} \int_{P \backslash\left(\cup_{k} D_{\varepsilon}\left(z_{k}\right) \cup \bigcup_{m} D_{\varepsilon}\left(\beta_{m}\right)\right)} \frac{K_{\bar{z}}}{F} d z \wedge d \bar{z}= \\
&-\frac{\sqrt{-1}}{2}\left(\int_{\partial P} \frac{K}{F} d z-\sum_{k} \int_{\partial D_{\varepsilon}\left(z_{k}\right)} \frac{K}{F} d z-\sum_{m} \int_{\partial D_{\varepsilon}\left(\beta_{m}\right)} \frac{K}{F} d z\right) .
\end{aligned}
$$

By the periodicities of $K$ and $F, \int_{\partial P} K / F d z=0$. On a neighborhood of $z_{k}$, $1 / F=\sigma d_{k} /\left(z-z_{k}\right)+g_{k}(z)$, where $g_{k}(z)$ is a function holomorphic on that neighborhood. Thus

$$
\int_{\partial D_{\varepsilon}\left(z_{k}\right)} \frac{K}{F} d z=\sigma d_{k} \int_{\partial D_{\varepsilon}\left(z_{k}\right)} \frac{K}{z-z_{k}} d z+\int_{\partial D_{\varepsilon}\left(z_{k}\right)} K g_{k}(z) d z .
$$

Since

$\lim _{\varepsilon \rightarrow 0} \int_{\partial D_{\varepsilon}\left(z_{k}\right)} K g_{k}(z) d z=0 \quad$ and $\quad \lim _{\varepsilon \rightarrow 0} \sigma d_{k} \int_{\partial D_{\varepsilon}\left(z_{k}\right)} \frac{K}{z-z_{k}} d z=\sigma d_{k} 2 \pi \sqrt{-1} K\left(z_{k}\right)$,

we have

$$
\lim _{\varepsilon \rightarrow 0} \int_{\partial D_{\varepsilon}\left(z_{k}\right)} \frac{K}{F} d z=\sigma d_{k} 2 \pi \sqrt{-1} K\left(z_{k}\right) .
$$

Similarly

$$
\lim _{\varepsilon \rightarrow 0} \int_{\partial D_{\varepsilon}\left(\beta_{m}\right)} \frac{K}{F} d z=\sigma d_{m}^{\prime} 2 \pi \sqrt{-1} K\left(\beta_{m}\right)
$$

Therefore,

$$
\begin{aligned}
A(g) & =-\pi \sigma\left(\sum_{k} d_{k} K\left(z_{k}\right)+\sum_{m} d_{m}^{\prime} K\left(\beta_{k}\right)\right) \\
& =-\pi \sigma \alpha_{\max }\left(K_{1}-K_{2}\right)=\pi\left(2 \alpha_{\max }-\alpha_{\min }\right) / K_{1} .
\end{aligned}
$$

This finishes the proof of Theorem 1.5.

The proof of the necessity component of Theorem 1.5 provides a blueprint for the explicit construction of an HCMU metric on $T^{2}$.

Theorem 4.1. Let $T^{2}=\mathbb{C} / \Gamma$ and $P_{\mathrm{o}}$ be the elementary parallelogram with base point the origin, with $p_{1}=z_{1}, \ldots, p_{n}=z_{n}$ in $P_{0}$. The real numbers $\alpha_{1}, \ldots, \alpha_{n}$ are positive, and $\alpha_{1}, \ldots, \alpha_{j}$ are integers with $\alpha_{i} \geq 2$ for $i=1, \ldots, j$. If there exists an HCMU metric on $T^{2}$ such that $z_{1}, \ldots, z_{j}$ are the saddle points of $K$, and $z_{j+1}, \ldots, z_{j+l}$ and $z_{j+l+1}, \ldots, z_{n}$ are respectively the maximum and minimum points of $K$, then the HCMU metric can be written in the form

$$
g=e^{2 \varphi}|d z|^{2} \quad \text { on } P_{\mathrm{o}} \backslash\left\{z_{1}, \ldots, z_{n}\right\},
$$


with $e^{2 \varphi}=-\frac{1}{3}\left(K-K_{1}\right)\left(K-K_{2}\right)\left(K+K_{1}+K_{2}\right) /|F|^{2}$, where $K$ is determined by the equation

$$
\begin{aligned}
& \left(K_{1}-K\right)\left(K-K_{2}\right)^{a-1}\left(K+K_{1}+K_{2}\right)^{-a} \\
& =A e^{\lambda z+\bar{\lambda} \bar{z}} \prod_{k=j+1}^{j+l}\left|\sigma\left(z-z_{k}\right)\right|^{2 \alpha_{k}} \prod_{k^{\prime}=j+l+1}^{n}\left|\sigma\left(z-z_{k^{\prime}}\right)\right|^{2(a-1) \alpha_{k^{\prime}}} \\
& \quad \times \prod_{m=1}^{t}\left|\sigma\left(z-\beta_{m}\right)\right|^{2} \prod_{m^{\prime}=t+1}^{s}\left|\sigma\left(z-\beta_{m^{\prime}}\right)\right|^{2(a-1)}
\end{aligned}
$$

on $P_{\mathrm{o}}$, and, on $P_{\mathrm{o}} \backslash\left\{z_{j+1}, \ldots, z_{n}, \beta_{1}, \ldots, \beta_{s}\right\}$,

$$
\begin{aligned}
\frac{1}{F}=\sigma\left(\lambda+\sum_{k=j+1}^{j+l} \alpha_{k} \zeta\left(z-z_{k}\right)\right. & +(a-1) \sum_{k^{\prime}=j+l+1}^{n} \alpha_{k^{\prime}} \zeta\left(z-z_{k^{\prime}}\right) \\
& \left.+\sum_{m=1}^{t} \zeta\left(z-\beta_{m}\right)+\sum_{m^{\prime}=t+1}^{s}(a-1) \zeta\left(z-\beta_{m^{\prime}}\right)\right) \\
= & \frac{B \prod_{i^{\prime}=1}^{j} \sigma\left(z-z_{i^{\prime}}\right)^{\left(\alpha_{i^{\prime}}-1\right)}}{\prod_{k=j+1}^{n} \sigma\left(z-z_{k}\right) \prod_{m=1}^{s-1} \sigma\left(z-\beta_{m}\right) \sigma\left(z-\beta_{s}-n_{1} w_{1}-n_{2} w_{2}\right)} .
\end{aligned}
$$

Here $K_{1}$ is the maximum of $K$ that is positive, $K_{2}$ is the minimum of $K$ and $K_{2} / K_{1}=\left(2 \alpha_{\min }-\alpha_{\max }\right) /\left(2 \alpha_{\max }-\alpha_{\min }\right)$, where $\alpha_{\max }=\alpha_{j+1}+\cdots+\alpha_{j+l}+t$, $\alpha_{\min }=\alpha_{j+l+1}+\cdots+\alpha_{n}+s-t$, and $a-1=-\alpha_{\max } / \alpha_{\min }$. Also $A$ and $B$ are constants, $s=\sum_{i^{\prime}=1}^{j} \alpha_{i^{\prime}}-n$ is the number of smooth extremal points of $K$, and $\beta_{1}, \ldots, \beta_{t}$ and $\beta_{t+1}, \ldots, \beta_{s}$ are respectively the smooth maximum and minimum points of $K$. Also $\lambda=1 /\left(w_{1} \bar{w}_{2}-w_{2} \bar{w}_{1}\right)\left(\operatorname{Re}\left(\eta_{1} \rho\right) \bar{w}_{2}-\operatorname{Re}\left(\eta_{2} \rho\right) \bar{w}_{1}\right)$ with $\eta_{1}=2 \zeta\left(w_{1} / 2\right)$ and $\eta_{2}=2 \zeta\left(w_{2} / 2\right)$. Finally

$$
\begin{aligned}
\rho & =2\left(\sum_{k=j+1}^{j+l} \alpha_{k} z_{k}+\sum_{k^{\prime}=j+l+1}^{n}(a-1) \alpha_{k^{\prime}} z_{k^{\prime}}+\sum_{m=1}^{t} \beta_{m}+\sum_{m^{\prime}=t+1}^{s}(a-1) \beta_{m^{\prime}}\right), \\
\sigma & =\frac{3}{\left(K_{2}-K_{1}\right)\left(2 K_{1}+K_{2}\right)},
\end{aligned}
$$

and $\sum_{i^{\prime}=1}^{j}\left(\alpha_{i^{\prime}}-1\right) z_{i^{\prime}}-\sum_{k=j+1}^{n} z_{k}-\sum_{m=1}^{s} \beta_{m}=n_{1} w_{1}+n_{2} w_{2}$.

Remark 4.1. By (48) and (67), the area formulas of HCMU metrics on $S^{2}$ and $T^{2}$ are the same. In fact in [Wu 2005] it has been proved that for any HCMU metric the area formula and the energy formula are the same:

$$
A(g)=\frac{\pi\left(2 \alpha_{\max }-\alpha_{\min }\right)}{K_{1}} \quad \text { and } \quad E(g)=\frac{\pi\left(\alpha_{\max }^{2}+\alpha_{\min }^{2}-\alpha_{\max } \alpha_{\min }\right) K_{1}}{2 \alpha_{\max }-\alpha_{\min }} .
$$


Finally, we raise a question: The formulas (11) and (14) share a formal similarity under the identifications of $1 /\left(z-z_{k}\right)$ with $\zeta\left(z-z_{k}\right)$ and $z-z_{i}$ with $\sigma\left(z-z_{i}\right)$. This leads us to speculate whether there exists a formula corresponding to (11) for a compact Riemannian surface of genus greater than one.

\section{Acknowledgments}

We would like to thank Professors X.H. Zhu and X.X. Chen for their helpful suggestions. Wu thanks Professors X.H. Zhu, X.X. Chen, C.K. Peng and W.Y. Ding for their enduring encouragement.

\section{References}

[Chen 2000] X. Chen, "Obstruction to the existence of metric whose curvature has umbilical Hessian in a K-surface”, Comm. Anal. Geom. 8:2 (2000), 267-299. MR 2001k:53060 Zbl 0971.53029

[Chen et al. 2005] Q. Chen, X. Chen, and Y. Wu, "The structure of HCMU metric in a $K$-surface", Int. Math. Res. Not. 16 (2005), 941-958. MR 2006f:53046

[Lang 1987] S. Lang, Elliptic functions, 2nd ed., Graduate Texts in Mathematics 112, Springer, New York, 1987. MR 88c:11028 Zbl 0615.14018

[Lin and Zhu 2002] C. S. Lin and X. Zhu, "Explicit construction of extremal Hermitian metrics with finite conical singularities on $S^{2 ",}$ Comm. Anal. Geom. 10:1 (2002), 177-216. MR 2003c:58010 Zbl 1021.58008

[McOwen 1988] R. C. McOwen, "Point singularities and conformal metrics on Riemann surfaces", Proc. Amer. Math. Soc. 103:1 (1988), 222-224. MR 89m:30089 Zbl 0657.30033

[Troyanov 1991] M. Troyanov, "Prescribing curvature on compact surfaces with conical singularities”, Trans. Amer. Math. Soc. 324:2 (1991), 793-821. MR 91h:53059 Zbl 0724.53023

[Wu 2005] Y. Wu, Some problems about HCMU metrics, thesis, University of Science and Technology of China, 2005.

Received November 29, 2007. Revised January 30, 2009.

\section{QING CHEN}

DePaRTMENT OF MATHEMATICS

UNIVERSity of SCIENCE AND TECHNOLOGY OF CHINA

HEFEI, ANHUI 230026

CHINA

qchen@ustc.edu.cn

\section{YINGYI WU}

SCHOOL OF MATHEMATICAL SCIENCES

Graduate University of The Chinese ACADEMy of SCIENCES

BEIJING 100049

CHINA

wuyy@gucas.ac.cn 